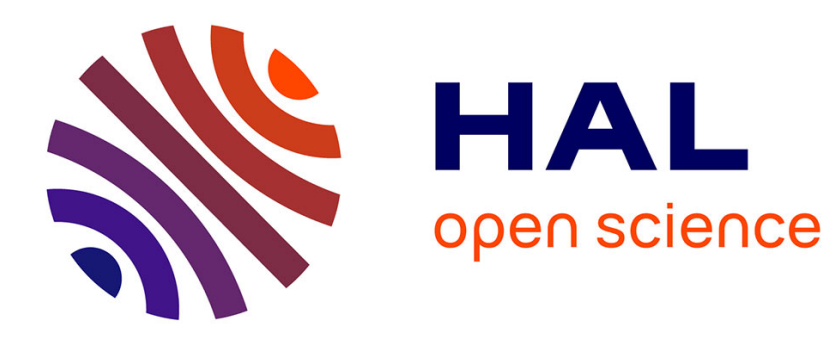

\title{
Analytic Combinatorics of Chord Diagrams
}

\author{
Philippe Flajolet, Marc Noy
}

\section{To cite this version:}

Philippe Flajolet, Marc Noy. Analytic Combinatorics of Chord Diagrams. [Research Report] RR-3914,

INRIA. 2000. inria-00072739

\section{HAL Id: inria-00072739 \\ https://hal.inria.fr/inria-00072739}

Submitted on 24 May 2006

HAL is a multi-disciplinary open access archive for the deposit and dissemination of scientific research documents, whether they are published or not. The documents may come from teaching and research institutions in France or abroad, or from public or private research centers.
L'archive ouverte pluridisciplinaire HAL, est destinée au dépôt et à la diffusion de documents scientifiques de niveau recherche, publiés ou non, émanant des établissements d'enseignement et de recherche français ou étrangers, des laboratoires publics ou privés. 


\title{
Analytic Combinatorics of Chord Diagrams
}

\author{
Philippe Flajolet, Marc Noy
}

$\mathrm{N}^{\circ} 3914$

March 2000

THÈME 2

\section{apport} derecherche 



\title{
Analytic Combinatorics of Chord Diagrams
}

\author{
Philippe Flajolet, Marc Noy \\ Thème 2 - Génie logiciel \\ et calcul symbolique \\ Projet Algo \\ Rapport de recherche $\mathrm{n}^{\circ} 3914$ - March 2000 - 11 pages
}

\begin{abstract}
In this paper we study the enumeration of diagrams of $n$ chords joining $2 n$ points on a circle in disjoint pairs. We establish limit laws for the following three parameters: number of components, size of the largest component, and number of crossings. We also find exact formulas for the moments of the distribution of number of components and number of crossings.
\end{abstract}

Key-words: Analytic combinatorics, chord diagram, asymptotic enumeration, limit distribution

(Résumé : tsvp)

Unité de recherche INRIA Rocquencourt

Domaine de Voluceau, Rocquencourt, BP 105, 78153 LE CHESNAY Cedex (France)

Téléphone : (33) 0139635511 - Télécopie : (33) 0139635330 


\section{Combinatoire analytique des diagrammes de cordes}

Résumé : Cet article est dédié au dénombrement de diagrammes de cordes reliant $2 n$ points sur un cercle par paires disjointes. On y établit les lois limites de trois paramètres: nombre de composantes connexes, taille de la plus grande composante, et nombre de croisements. Des formules exactes pour les moments du nombre de composantes et du nombre de croisements sont aussi données.

Mots-clé : Combinatoire analytique, diagramme de cordes, dénombrement asymptotique, loi limite. 


\title{
Analytic Combinatorics of Chord Diagrams
}

\author{
Philippe Flajolet \\ INRIA Rocquencourt \\ Marc Noy \\ UPC Barcelona
}

March 4, 2000

\begin{abstract}
In this paper we study the enumeration of diagrams of $n$ chords joining $2 n$ points on a circle in disjoint pairs. We establish limit laws for the following three parameters: number of components, size of the largest component, and number of crossings. We also find exact formulas for the moments of the distribution of number of components and number of crossings.
\end{abstract}

\section{Introduction}

Take $2 n$ points on a circle labeled $1,2, \ldots, 2 n$ and join them in disjoint pairs by $n$ chords. The resulting configuration is called a chord diagram (see Fig. 1). Besides its intrinsic combinatorial interest, the enumeration of chord diagrams is relevant to other fields, like the analysis of data structures in computer science [4,5], or the study of invariants in knot theory [18].

A classical result is that the number of diagrams were the chords do not cross is a Catalan number. In a series of papers Touchard studied the problem of counting diagrams according to the number of crossings of the chords and found the corresponding generating function in the form of a continued fraction [19]. A remarkable exact formula in terms of the ballot numbers was implicit in the work of Touchard and made explicit later by Riordan [14]. This formula was revisited in $[13,10,12]$.

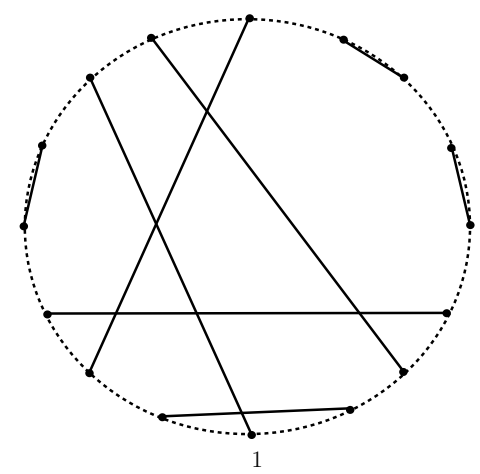

Figure 1: A chord diagram with 4 components.

Touchard also considered connected diagrams, that is, diagrams where no set of chords can be separated from the remaining chords by a line. A recurrence relation for the number of 
connected diagrams was found by Stein [16]. At the same time Stein and Everett [17] found an asymptotic expression for this number. If we define a component in a diagram as a maximal connected subdiagram, a natural extension is to study the enumeration of diagrams according to the number of components, a problem for which we have not found explicit references.

In this paper we reexamine these problems and we analyze chord diagrams according to several basic parameters: the number of components, the size of the largest component, and the number of crossings, deriving limit laws for the corresponding distributions. For the first two parameters we show in Section 1 that the limit laws are given by a modification of a Poisson distribution of parameter 1 . We also find exact formulas for the mean and the variance of the number of components. In the case of the number of crossings, we show in Section 2 that the distribution is asymptotically Gaussian. The main tool in establishing this result is an integral representation of the corresponding generating function. This integral representation also allows the computation of the moments of any fixed order.

Our analysis is based on general methods of analytic combinatorics [9, 15]. First we find specifications of combinatorial structures that translate into symbolic generating functions (GF). Then we apply analytic methods to obtain asymptotic estimates and derive limit laws. This work can be considered as a counterpart to a previous paper of the authors [6], where we analyzed non-crossing configurations on the vertices of a convex polygon. Whereas in the non-crossing case the GFs involved were algebraic, the present situation is quite different as we are dealing with divergent series.

Throughout the paper the size of a diagram will be the number of chords. By slightly perturbing the points if necessary, we can assume that no three chords in a diagram intersect in a point.

\section{Components}

If we let $I_{n}$ be the number of all diagrams of size $n$, then it is well known that

$$
I_{n}=1 \cdot 3 \cdot 5 \cdots(2 n-1)=\frac{(2 n) !}{2^{n} n !} .
$$

Let

$$
I(z)=\sum_{n \geq 0} I_{n} z^{n}
$$

be the corresponding ordinary generating function. Note that we operate here with ordinary GFs having radius of convergence 0 .

Let $C_{n}$ be the number of connected diagrams of size $n$ and let $C(z)=\sum C_{n} z^{n}$ be the corresponding OGF. The basic relation is

$$
I(z)=1+C\left(z I(z)^{2}\right)
$$

and it is proved as follows. In a diagram, the particular connected component determined by containing vertex 1 is called the root component. In the intervals defined by the edges, arbitrary diagrams may be inserted. If the root has $j$ chords, then it defines $2 j$ spacings. Thus, $I(z)=1+\sum C_{n} z^{n} I(z)^{2 n}=1+C\left(z I(z)^{2}\right)$, where the term 1 represents the empty diagram. This gives a recursive determination of $C(z)$,

$$
C(z)=z+z^{2}+4 z^{3}+27 z^{4}+248 z^{5}+2830 z^{6}+38232 z^{7}+593859 z^{8}+\cdots .
$$

Let now $I_{n, k}$ be the number of diagrams of size $n$ and exactly $k$ components, and let

$$
I(z, w)=\sum I_{n, k} z^{n} w^{k}
$$


be the corresponding bivariate GF. As with equation (1) we have

$$
I(z, w)=1+w C\left(z I(z, w)^{2}\right),
$$

where the factor $w$ marks the component containing vertex 1 . From the known expansion of $C(z)$ we get

$$
I(z, w)=1+w z+\left(w+2 w^{2}\right) z^{2}+\left(4 w+6 w^{2}+5 w^{3}\right) z^{3}+\left(27 w+36 w^{2}+28 w^{3}+14 w^{4}\right) z^{4}+\cdots
$$

In order to express our first result we define the numbers $h_{n}$ by means of

$$
\frac{1}{I(z)}=H(z)=\sum h_{n} z^{n} \text {. }
$$

Theorem 1 Let $X_{n}$ be the number of components in a random diagram of size $n$.

(i) For $k \geq 1$, one has

$$
\operatorname{Pr}\left\{X_{n}=k\right\}=e^{-1} \cdot \frac{1}{(k-1) !}(1+o(1)) \quad n \rightarrow \infty .
$$

In other words, $-1+X_{n}$ is distributed like a Poisson law of parameter 1.

(ii) The mean and the variance of the distribution of $X_{n}$ are

$$
\begin{aligned}
\mu_{n} & =\frac{I_{n+1}+h_{n+1}}{I_{n}} \sim 2, \\
\sigma_{n}^{2} & =\frac{I_{n+2}-2 I_{n+1}+(4 n+1) h_{n+1}-2 n(2 n-1) h_{n}}{I_{n}}-\mu_{n}^{2} \sim 1,
\end{aligned}
$$

where the numbers $h_{n}$ are defined in (3).

Proof. (i) A diagram is said to be "monolithic" if: (1) it consists solely of the connected component that contains 1 (called the root component) and of isolated edges; (2) for any two such isolated edges $(a, b)$ and $(c, d)$, one never has $a<c<d<b$ nor $c<a<b<d$ (for instance, the diagram in Fig. 1 is a monolith).

The OGF of monoliths is clearly

$$
M(z)=C\left(\frac{z}{(1-z)^{2}}\right)
$$

since we can accommodate any sequence of isolated edges in each "leg" of the root component as well as after the root component. Then clearly,

$$
M_{n}=C_{n}+\left(\begin{array}{c}
2 n-2 \\
1
\end{array}\right) C_{n-1}+\left(\begin{array}{c}
2 n-3 \\
2
\end{array}\right) C_{n-2}+\left(\begin{array}{c}
2 n-4 \\
3
\end{array}\right) C_{n-3}+\cdots .
$$

According to Stein and Everett [17] we have

$$
\frac{C_{n}}{I_{n}}=e^{-1}+o(1)
$$

and we deduce

$$
M_{n} \sim e^{-1}\left(1+\frac{1}{1 !}+\frac{1}{2 !}+\frac{1}{3 !} \cdots\right) \sim I_{n} .
$$

Thus, the probability that a random diagram is a monolith tends to 1 , as $n \rightarrow \infty$, a result already observed in [17]. Asymptotically, it suffices to analyze the number of components in a 
monolith. The number of monoliths with $k$ components is the same as the number of monoliths in which the root component has size $n-k+1$, since every other component is an isolated edge. But this is

$$
C_{n-k+1}\left(\begin{array}{c}
2 n-k \\
k-1
\end{array}\right) \sim I_{n} \cdot e^{-1} \frac{1}{(k-1) !} .
$$

(ii) In order to compute $E\left\{X_{n}\right\}$ and $E\left\{X_{n}^{2}\right\}$ we need to evaluate $\frac{\partial}{\partial w} I(z, w)$ and $\frac{\partial^{2}}{\partial w^{2}} I(z, w)$ at $w=1$ (see [15]). According to Stein [16] the numbers $C_{n}$ satisfy the recurrence relation

$$
C_{n}=(n-1) \sum_{j=1}^{n-1} C_{j} C_{n-j}, \quad C_{1}=1,
$$

(see [11] for a simpler proof of this result) and this in turn implies that the GF satisfies the following formal differential equation:

$$
2 z C(z) \frac{d C(z)}{d z}=C(z)^{2}+C(z)-z .
$$

Differentiating (2) with respect to $w$, using (4) and eliminating $C(z)$ we get

$$
\left.\frac{\partial}{\partial w} I(z, w)\right|_{w=1}=\frac{1}{z}\left(I(z)+\frac{1}{I(z)}-2\right) .
$$

Similarly, one obtains

$$
\left.\frac{\partial^{2}}{\partial w^{2}} I(z, w)\right|_{w=1}=\frac{(I(z)-1)^{2}\left(I(z)+(1-3 z) I(z)^{2}-2\right)}{z^{2} I(z)} .
$$

Expanding the last two series we get the formulas stated for $\mu_{n}$ and $\sigma_{n}^{2}$.

The asymptotic values of $\mu_{n}$ and $\sigma_{n}^{2}$ are to be expected in view of part (i), but they cannot be guaranteed just from pointwise convergence to the Poisson distribution. However, they can can be found as follows (see [2, p. 294, ex. 16] for a similar problem). If we let $g_{n}=h_{n} / I_{n}$, then from (3) we have

$$
g_{n}=1-\sum_{k=1}^{n-1} g_{k}\left(\begin{array}{l}
n \\
k
\end{array}\right)\left(\begin{array}{l}
2 n \\
2 k
\end{array}\right)^{-1},
$$

and from this it follows easily that $g_{n}=1+O\left(n^{-1}\right)$. The values $g_{1}=1$ and $g_{2}=2 / 3$ give a more precise estimate

$$
g_{n}=1-\frac{1}{n}+\frac{3}{4 n^{2}}+O\left(n^{-3}\right)
$$

which is enough for our purposes. Indeed, we have

$$
\mu_{n}=\frac{I_{n+1}+h_{n+1}}{I_{n}}=\frac{2 n+1}{n+1}+O\left(n^{-1}\right) \sim 2 .
$$

and similarly for the variance.

Note. It is a curiosity that the same shifted Poisson law appears in many "subcritical" set constructions [9, Ch. 9]; for instance it is asymptotically the distribution of the root degree of a random Cayley tree.

More can be said about the number of components. Let $I_{k}(z)=\left[w^{k}\right] I(z, w)$ be the GF of diagrams with $k$ components. Then, applying the Lagrange inversion theorem to equation (2), we get

$$
I_{k}(z)=\frac{1}{k}\left[u^{k-1}\right] C\left(z(1+u)^{2}\right)^{k} .
$$


(Set $I=1+J$ to recover a classical case of application of Lagrange inversion.) Now, apply Taylor's formula to $C$. This gives

$$
C\left(z(1+u)^{2}\right)=C(z+z u(2+u))=\sum_{j=0}^{\infty} \frac{1}{j !} \frac{\partial^{j} C(z)}{\partial z^{j}} z^{j} u^{j}(2+u)^{j}
$$

Thus, when raising this expression to the $k$-th power and upon extracting coefficients of $u^{k-1}$, one finds that: Each $I_{k}(z)$ is a polynomial in $C(z)$ and its derivatives. For instance, we have

$$
\begin{aligned}
& I_{1}(z)=C(z) \\
& \left.I_{2}(z)=2 z C(z) C^{\prime}(z)\right) \\
& I_{3}(z)=z C(z)^{2} C^{\prime}(z)+2 z^{2} C(z)^{2} C^{\prime \prime}(z)+4 z^{2} C(z) C^{\prime}(z)^{2}
\end{aligned}
$$

In other words, for each $I_{k}$ there is a polynomial $P_{k}\left(y_{0}, y_{1}, y_{2}, \ldots\right)$ (a sort of Bell polynomial) such that

$$
I_{k}(z)=P_{k}\left(C, \theta C, \theta^{2} C, \ldots\right)
$$

where $\theta \equiv z \frac{d}{d z}$.

Largest connected component. The second parameter we analyze in this section is the size of the largest component in a random diagram. The answer comes again by considering monoliths.

Theorem 2 Let $L_{n}$ be the size of largest connected component in a random diagram of size $n$. Then,

$$
E\left\{L_{n}\right\}=n-1+o(1), \quad \operatorname{Var}\left\{L_{n}\right\}=1+o(1),
$$

and for any fixed $k \geq 1$, one has

$$
\operatorname{Pr}\left\{n-L_{n}=k\right\}=e^{-1} \cdot \frac{1}{k !}(1+o(1)) \quad n \rightarrow \infty .
$$

In other words, $n-L_{n}$ is distributed like a Poisson law of parameter 1.

Proof. Asymptotically, it suffices to analyse the largest component in a monolith. In fact, it suffices to analyse the size of the root component.

The same analysis as in the proof of Theorem 1 shows that the number of monoliths of size $n$ with root component of size $n-k$ is given by

$$
M_{n, k}=C_{n-k}\left(\begin{array}{c}
2 n-k-1 \\
k
\end{array}\right) \sim I_{n} \cdot e^{-1} \frac{1}{k !} .
$$

This proves that $n$ minus the size of the root component of a random monolith is asymptotically Poisson(1). As monoliths asymptotically exhaust all cases, this establishes the analogous properties for random diagrams. This root component is obviously the largest component with probability $1-o(1)$, so that we are done.

Note. All this gives a precise probabilistic picture of a random diagram: It consists of the root component (the component of 1) that is almost surely the largest component and a few isolated edges, with additionally no two isolated edges entering a dominance relation. (In other words, the associated tree in the tree decomposition is of height equal to 2 with high probability.) Consideration of

$$
M^{\star}(z)=C\left(z(1+z)^{2}\right),
$$

the OGF of monoliths where no leg has more than one isolated edge, shows that almost surely, there are $O(1)$ isolated edges, all found in different legs of the root component. 


\section{Crossings}

Let $\kappa$ denote the number of chord crossings in a chord diagram, and let $\mathcal{I}_{n}$ be the set of all diagrams of size $n$. For a diagram in $\mathcal{I}_{n}$, the parameter $\kappa$ lies between 0 and $n(n-1) / 2$. A remarkable exact formula was discovered by Touchard and Riordan, namely

$$
\phi_{n}(q):=\sum_{w \in \mathcal{I}_{n}} q^{\kappa(w)}=\frac{1}{(1-q)^{n}} \sum_{k=-n}^{n}(-1)^{k} q^{k(k-1) / 2}\left(\begin{array}{c}
2 n \\
n+k
\end{array}\right) .
$$

¿From the combinatorial origin of the problem, we know that $\phi_{n}(q)$ is a polynomial in $q$ with degree $n(n-1) / 2$. The formula (5) involves heavy cancellations so that it is far from obvious given only the analytic form of $\phi_{n}$ that it must be a polynomial, nor even that $\phi_{n}(1)=$ $1 \cdot 3 \cdots(2 n-1)$. The moments of the distribution are given by the values of the derivatives $\phi^{(r)}(1)$. Again, though they are "contained" in the Touchard-Riordan formula, it is not $a$ priori clear whether they admit a simple form.

We prove here:

Theorem 3 Let $X_{n}$ be the random variable equal to the value of $\kappa$ taken over the set of chord diagrams $\mathcal{I}_{n}$ of size $n$ endowed with the uniform probability distribution.

(i) The mean and the variance of the distribution of $X_{n}$ are

$$
\mu_{n}:=E\left\{X_{n}\right\}=\frac{n(n-1)}{6}, \quad \sigma_{n}^{2}:=\operatorname{Var}\left\{X_{n}\right\}=\frac{n(n-1)(n+3)}{45},
$$

(ii) The distribution of $X_{n}$ is Gaussian in the asymptotic limit: for all real x, one has

$$
\lim _{n \rightarrow \infty} \operatorname{Pr}\left\{\frac{X_{n}-\mu_{n}}{\sigma_{n}} \leq x\right\}=\frac{1}{\sqrt{2 \pi}} \int_{-\infty}^{x} e^{-y^{2} / 2} d y .
$$

Proof. (i) The exact values of the mean and the variance are mentioned by Riordan [14] who says that he "does not take place to prove the results". A proof was subsequently given by Flajolet, Puech and Vuillemin [7] using orthogonality properties of $q$-Hermite polynomials.

A direct proof of Riordan's mean value result can be based on the decomposition of the number of crossings as

$$
X_{n}=\sum_{1 \leq a<b<c<d \leq 2 n} Y(a, b, c, d),
$$

where $Y(a, b, c, d)$ is a random variable whose value is 1 if the number of edges attached to $a, b, c, d$ is exactly 2 and these edges cross, the value of $Y$ being 0 otherwise. By symmetry, the mean of each $Y$ conditioned upon the fact that $a, b, c, d$ define two edges exactly is $1 / 3$; the mean value $E\left\{X_{n}\right\}=\frac{1}{3} \frac{n(n-1)}{2}$ results from there. The extension of this approach to a determination of the variance is practicable but the combinatorics for higher moments soon becomes intractable.

(ii) A different approach to moment determinations is proposed here that also leads to the determination of the limit distribution. The heart of the matter is the following integral representation of certain $q$-series that Flajolet and Salvy [8] have used in the enumeration of connected graphs by excess of number of edges over number of nodes.

Lemma 4 Let $u_{k}$ be a number sequence with ordinary generating function $U(z)=\sum_{k} u_{k} z^{k}$ that is assumed to be a Laurent polynomial. Then the q-sum

$$
S(q):=\sum_{k} q^{k^{2} / 2} u_{k}
$$


admits the integral representation

$$
S\left(e^{t}\right)=\frac{1}{\sqrt{2 \pi}} \int_{-\infty}^{+\infty} e^{-x^{2} / 2} U\left(e^{x \sqrt{t}}\right) d x
$$

Proof. Start from the Gaussian integral

$$
\int_{-\infty}^{+\infty} e^{-x^{2} / 2} d x=\sqrt{2 \pi}
$$

then set $x \mapsto x-a$ to get

$$
e^{a^{2} / 2}=\frac{1}{\sqrt{2 \pi}} \int_{-\infty}^{+\infty} e^{-x^{2} / 2} e^{a x} d x
$$

The lemma is obtained upon setting $a \mapsto k \sqrt{t}$ and summing.

The formula (6) "linearizes" the exponent in a quantity like $q^{a^{2} / 2}$, which constitutes the real strength of the method (various other conditions may of course be imposed on $U(z)$ ). For the generating function of crossings, we have $u_{k}=(-1)^{k}\left(\begin{array}{c}2 n \\ n+k\end{array}\right) q^{-k / 2}$, the generating function is

$$
U(z)=(-1)^{n} q^{-n / 2}(\sqrt{z}-\sqrt{q / z})^{2 n},
$$

and application of the lemma yields

$$
\phi_{n}\left(e^{t}\right)=\frac{1}{\sqrt{2 \pi}} \int_{-\infty}^{+\infty} e^{-x^{2} / 2} x^{2 n} H(x, t)^{n} d x
$$

where

$$
H(x, t)=\frac{2 \sinh ^{2}(x \sqrt{t} / 2-t / 4)}{x^{2} \exp (t / 2) \sinh (t / 2)} .
$$

This formula is enough to derive the number of diagrams (!) by taking the limit as $t \rightarrow 0$,

$$
\phi_{n}(1)=\lim _{t \rightarrow 0} \phi_{n}\left(e^{t}\right)=\frac{1}{\sqrt{2 \pi}} \int_{-\infty}^{\infty} e^{-x^{2} / 2} x^{2 n} d x=\frac{(2 n) !}{2^{n} n !} .
$$

Differentiation with respect to $t$ followed by passage to the limit $t \rightarrow 0$ gives access mechanically to moments of any fixed order. The process is well within the capabilities of a computer algebra system like Maple, and one finds in this way:

$$
\begin{aligned}
& E\left\{X_{n}^{0}\right\}=1 \\
& E\left\{X_{n}^{1}\right\}=\frac{1}{6} n(n-1) \\
& E\left\{X_{n}^{2}\right\}=\frac{1}{180} n(n-1)\left(5 n^{2}-n+12\right) \\
& E\left\{X_{n}^{3}\right\}=\frac{1}{7560} n(n-1)\left(35 n^{4}+14 n^{3}+235 n^{2}-188 n+24\right)
\end{aligned}
$$

Notice that the formulas for the mean and the variance follow directly from the above expressions.

The limit distribution is next obtained by showing that the Laplace transform of a standardized version of $X_{n}$ converges pointwise to the Laplace transform of a normal variable. By the continuity theorem for Laplace transforms [1], this entails convergence in law to a normal variable. We thus set

$$
X_{n}^{\star}=\frac{X_{n}-\mu_{n}}{\sigma_{n}}, \quad \psi(u)=E\left\{e^{u X_{n}^{\star}}\right\}
$$


so that

$$
\psi(u)=e^{-u \mu_{n} / \sigma_{n}} \frac{\phi_{n}\left(e^{u / \sigma_{n}}\right)}{\phi_{n}(1)} .
$$

When $u$ is a fixed real number, $\psi(u)$ as provided by $(7),(8),(10)$, can be evaluated through Laplace's method [3]. The case of $\psi(0)$ is a textbook example of the method. The maximum of the integrand is attained at $x= \pm \zeta$, where $\zeta=\sqrt{2 n}$, and the contribution of the integral outside an interval of length $2 \log n$ around the points $\pm \zeta$ is exponentially small, being of the form $e^{-c \log ^{2} n}$, for some $c>0$. It then suffices to establish the corresponding property when $u \neq 0$. We do this for $u>0$ and examine the positive part of the real line, the other cases being similar.

In the range $x \geq 2 \zeta$, the quantity $e^{-x^{2} / 2} x^{2 n} H^{n}$ decreases (its derivative is negative for large enough $n$ ), while being already exponentially small at $2 \zeta$. By the fast decrease of $e^{-x^{2} / 2}$, the contribution of the integrand for $x \geq 2 \zeta$ is therefore exponentially small. Thus, for dominant asymptotics, we may well restrict a priori the integration interval to $[-2 \zeta, 2 \zeta]$. But, in this domain, the quantity $H(x, t)$ is readily evaluated by Taylor expansions at 0 since $t=O\left(n^{-3 / 2}\right)$ and $x=O(\sqrt{n})$.

Setting $x=\zeta+y$ with the additional restriction $|y| \leq \log n$, we find

$$
n \log H\left(\sqrt{2 n}+y, \frac{u}{\sigma_{n}}\right)=u \frac{\sqrt{5 n}}{2}+\sqrt{\frac{5}{2}} u y-\frac{1}{8} u^{2}+O\left(n^{-1 / 5}\right) .
$$

It then suffices to integrate $H^{n}$ against $e^{-x^{2} / 2} x^{2 n}$ for $|y| \leq \log n$ (by Equations (7), (8)), complete the tails, and multiply by the normalization factor $\exp \left(-u \mu_{n} / \sigma_{n}\right)$ from (10). This purely mechanical process (intermediate computations omitted) yields

$$
e^{-u \mu_{n} / \sigma_{n}} \phi\left(e^{u / \sigma_{n}}\right)=e^{-n} n^{2 n} \int_{-\infty}^{+\infty} \exp \left(-y^{2}+\sqrt{\frac{5}{2}} u y-\frac{u^{2}}{8}\right) d y \cdot\left(1+O\left(n^{-1 / 5}\right)\right) .
$$

The contribution arising from the peak of the integrand at $-\zeta$ is similar, and one finds

$$
\psi(u)=e^{u^{2} / 2}\left(1+O\left(n^{-1 / 5}\right)\right) .
$$

Thus, the Laplace transform of the distribution converges pointwise to that of a standard normal variable, and the limit law is established.

Note. The evaluations of (9) hide nontrivial and somewhat mysterious identities starting with the Touchard-Riordan identity

$$
1 \cdot 3 \cdot 5 \cdots(2 n-1)=\sum_{k=-n}^{n}(-1)^{k}\left(\begin{array}{c}
\frac{k(k-1)}{2} \\
n
\end{array}\right)\left(\begin{array}{c}
2 n \\
n+k
\end{array}\right) .
$$

More generally, there is a computable polynomial $P_{r}(n)$ such that

$$
(1 \cdot 3 \cdot 5 \cdots(2 n-1)) \cdot P_{r}(n)=\sum_{k=-n}^{n}(-1)^{k}\left(\begin{array}{c}
\frac{k(k-1)}{2} \\
n+r
\end{array}\right)\left(\begin{array}{c}
2 n \\
n+k
\end{array}\right) .
$$

\section{References}

[1] P. Billingsley, Probability and measure, third edition, John Wiley \& Sons (1995). 
[2] L. Comtet, Advanced Combinatorics, Reidel (1974).

[3] N.G. De Bruijn, Asymptotic Methods in Analysis, Dover (1981).

[4] P. Flajolet, Combinatorial aspects of continued fractions, Discrete Math. 32 (1980), 125161.

[5] P. Flajolet, J. Françon and J. Vuillemin, Sequence of operations analysis for dynamic data structures, J. Algorithms 1 (1980), 111-141.

[6] P. Flajolet and M. Noy, Analytic Combinatorics of Non-crossing Configurations, Discrete Mathematics 204 (1999), 203-229.

[7] P. Flajolet, C. Puech and J. Vuillemin, The analysis of simple list structures, Inform. Sci. 38 (1986), 121-146.

[8] P. Flajolet and B. Salvy, unpublished manuscript.

[9] P. Flajolet and R. Sedgewick, Analytic Combinatorics, book in preparation (individual chapters are available as INRIA Research Reports 1888, 2026, 2376, 2956, 3162).

[10] M.E.H. Ismail, D. Stanton and G. Viennot, The combinatorics of $q$-Hermite polynomials and the Askey-Wilson integral, European J. Combin. 8 (1987), 379-392.

[11] A. Nijenhuis and H.S. Wilf, The Enumeration of Connected Graphs and Linked Diagrams, J. Combinatorial Theory A 27 (1979), 356-359.

[12] J.-G. Penaud, Une preuve bijective d'une formule de Touchard-Riordan, Discrete Mathematics 139 (1995), 347-360.

[13] R.C. Read, The chord intersection problem, Annals of the New York Academy of Sciences 139 (1979), 444-454.

[14] J. Riordan, The Distribution of Crossings of Chords Joining Pairs of $2 n$ points on a Circle, Math. of Computation 29 (1975), 215-222.

[15] R. Sedgewick and P. Flajolet, An introduction to the analysis of algorithms, AddisonWesley (1996).

[16] P.R. Stein, On a Class of Linked Diagrams, I. Enumeration, J. of Combinatorial Theory A 24 (1978), 357-366.

[17] P.R. Stein and C.J. Everett, On a Class of Linked Diagrams, II. Asymptotics, Discrete Mathematics 21 (1978), 309-318.

[18] A. Stoimenow, Enumeration of chord diagrams and an upper bound for Vassiliev invariants, J. Knot Theory Ramifications 7 (1998), 93-114.

[19] J. Touchard, Sur un problème de configurations et sur les fractions continues, Canad. J. Math. 4 (1952), 2-25. 


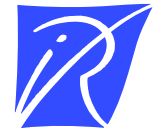

Unité de recherche INRIA Lorraine, Technopôle de Nancy-Brabois, Campus scientifique, 615 rue du Jardin Botanique, BP 101, 54600 VILLERS LÈS NANCY

Unité de recherche INRIA Rennes, Irisa, Campus universitaire de Beaulieu, 35042 RENNES Cedex Unité de recherche INRIA Rhône-Alpes, 655, avenue de l'Europe, 38330 MONTBONNOT ST MARTIN

Unité de recherche INRIA Rocquencourt, Domaine de Voluceau, Rocquencourt, BP 105, 78153 LE CHESNAY Cedex

Unité de recherche INRIA Sophia-Antipolis, 2004 route des Lucioles, BP 93, 06902 SOPHIA-ANTIPOLIS Cedex

Éditeur

INRIA, Domaine de Voluceau, Rocquencourt, BP 105, 78153 LE CHESNAY Cedex

(France)

http://www.inria.fr

ISSN 0249-6399 